\title{
Multirate Multiservice All-Optical Code Switched GMPLS Core Network Utilizing Multicode Variable- Weight Optical Code-Division Multiplexing
}

\author{
Ahmed E. Farghal, Hossam M. H. Shalaby, and Zen Kawasaki
}

\begin{abstract}
A multicode variable-weight (MCVW) technique is proposed for generalized multiprotocol label switching (GMPLS) optical networks in order to support multirate and integrated multimedia services. Under this technique, the number of simultaneously assigned codewords to each user is a function of the data rate of the service class, while quality-of-service differentiation is achieved using variable-weight codewords for each service class. The traffic behavior of the network is modeled using a multiservice loss model, and the probability density functions of the number of busy codes in the fiber link are obtained by the Kaufman-Roberts algorithm. In order to analyze the performance of the proposed multiservice multicode GMPLS optical network, several measures are derived and investigated, specifically, the bit-error rate, probability of degradation, blocking probability, and steady-state throughput. These performance measures are obtained for two different receiver structures, namely, correlation receivers with and without hard limiters. The performance of our optical GMPLS network, based on the multicode switching path, is compared with that of traditional optical GMPLS networks, based on the label switching path. The results show the superiority of the proposed technique when compared to traditional ones.
\end{abstract}

Index Terms-Generalized multiprotocol label switching (GMPLS); Multicode switching path (MCSP); Multirate; Optical code-division multiplexing (OCDM); Optical networks; Quality of service (QoS); Throughput.

\section{INTRODUCTION}

D riven by the insatiable demand for bandwidth increase, as a consequence of the emergence of

Manuscript received December 4, 2013; revised April 8, 2014; accepted June 11, 2014; published July 14, 2014 (Doc. ID 201977).

A. E. Farghal (e-mail: ahmed.farghal@ejust.edu.eg) is with the Department of Electronics and Communications Engineering, Egypt-Japan University of Science and Technology (E-JUST), Alexandria 21934, Egypt.

H. M. H. Shalaby is with the Department of Electronics and Communications Engineering, Egypt-Japan University of Science and Technology (E-JUST), Alexandria 21934, Egypt, and is on leave from the Electrical Engineering Department, Alexandria University, Alexandria 21544, Egypt.

Z. Kawasaki is with the Graduate School of Engineering, Osaka University, Osaka 565-0871, Japan.

http://dx.doi.org/10.1364/JOCN.6.000670 multimedia applications, optical networks are the superior candidates to support huge bandwidth as well as diverse service demands in a cost-effective manner. Wavelengthdivision multiplexing (WDM) techniques provide platforms to exploit the potential huge capacity of the fiber-optic transmission medium by simultaneously transmitting data on multiple wavelengths on a single fiber. Due to optical-electrical-optical (OEO) conversion at every switching or routing node, WDM capability is not completely exploited in a whole network due to the slowness of data processing in the electronic domain. All-optical networks (AONs), e.g., optical circuit switching (OCS), optical burst switching (OBS), and optical packet switching (OPS) networks in which data processing is carried entirely in the optical domain without OEO conversion, have been proposed to cope with the electronic switching bottleneck. OPS is viewed as the most promising technology in future $\mathrm{AONs}$, due to its characteristics of practical implementation of IPs in the optical domain, high-speed data transmission, data rate/format transparency, power efficiency, fine granularity, and flexibility [1].

One of the most efficient protocols, proposed for future $\mathrm{AONs}$, is generalized multiprotocol label switching (GMPLS) [2,3]. GMPLS protocols are connection-oriented control-plane protocols for setting up label switching paths (LSPs) that are identified by labels. A GMPLS protocol extends multiprotocol label switching (MPLS) to support devices that perform packet switching, time-division multiplexing (TDM), wavelength $(\lambda)$ switching, waveband switching, and fiber switching. GMPLS protocols offer effective network resource management and traffic engineering (constrained based routing), and simplify the network implementation as a whole $[\underline{4}, \underline{5}]$.

Utilizing optical code-division multiplexing (OCDM) technology for data switching in optical core networks has been discussed in [ $\underline{6}-\underline{9}]$, where the code of the received optical data is considered as a label. Advantages such as asynchronous accessibility [10], distributed control, and differentiated services with quality of service (QoS) at the physical layer have made OCDM rather attractive toward all-optical communications. Moreover, OCDM 
provides more label space and fine subwavelength data granularity for GMPLS networks. Two approaches, optical code (OC)-labeled path and OCDM path, have been studied [6]. In OC-labeled path, an OC label is only attached to the head of a packet. This limits the throughput because the packets on the same wavelength have to be processed serially. In OCDM path, the whole data packet including the header is encoded using a distinct OC. OCDM paths can be multiplexed onto the same wavelength and enable the router to process packets in parallel, which results in a simple architecture with no need for optical buffers. Furthermore, hybrid WDM/OCDM-based routing, in which both the wavelength and the OC of packets $(\lambda, C)$ are considered as the identifying label, has been studied in [․ㅡ, $]$.

Future optical networks are expected to provide broadband access to a high number of users with very different transmission rates and QoS traffic requirements. Recently, optical orthogonal frequency division multiplexing (OFDM)-based elastic optical network architecture with flexible data rate and spectrum allocation called spectrum-sliced elastic optical path (SLICE) was proposed in [11]. SLICE architecture can provide fine-granularity capacity to connections by elastically allocating spectrum using a variable number of low-rate OFDM subcarriers according to the transmitted data rate.

Various techniques have been proposed to support multirate services provision using OCDM, e.g., varying the code length [12,13], adopting optical fast frequency hopping [14], and adopting multicode-keying (MK) schemes [15, 16$]$.

A different approach to provide data rate differentiation is to use multicode (MC) techniques, in which a data rate differentiation is performed by assigning a set of codes according to the required data rate of each service class [17-20]. Due to the fixed code length of different classes, the system performance is not degraded. However, because of the need for transmitting many codes simultaneously, the number of supported users in the MC technique is decreased, which limits its applicability. Code families with large cardinalities, e.g., two-dimensional (2D) wavelengthhopping time-spreading (WHTS) codes, are required in the MC scheme. Hybrid OCDM/WDM is an alternative technique to resolve the problem of limited codes, where every code is reusable and can be sent simultaneously at different wavelengths [21]. The bit-error rate (BER) at the receiver side can be controlled using variable-weight (VW) optical codes, providing a provision of QoS differentiation in OCDM networks [22].

In this paper we propose to use multicode variableweight optical-orthogonal codes (MCVW-OOCs) for supporting multirate and multi-QoS transmission and providing path switching in optical GMPLS networks, where different service classes are accommodated. We refer to the path switching and GMPLS network based on the MC scheme as the multicode switching path (MCSP) and optical multicode switching-GMPLS (OMCS-GMPLS) network, respectively. The OMCS-GMPLS network provides the support of various data rates and multi-QoS by elastically assigning a number of fixed length codewords and choosing an appropriate code weight according to the connection demands. In the proposed network, we consider traffic with a fixed bandwidth and service requirements case, while the case of traffic that, upon arrival, may have different possible bandwidth requirements depending on the bandwidth availability will be investigated in future studies.

In order to investigate the OMCS-GMPLS network performance, three important performance metrics are derived for correlation receivers with and without hard limiters, which are the blocking probability, the BER, and the steady-state throughput. The effects of the number of simultaneous active users, user activity, and offered traffic loads on these performance metrics are also investigated.

The rest of this paper is organized as follows. Section II is devoted to the architecture and the operation of the proposed OMCS-GMPLS network. The performance of the OMCS-GMPLS network is analyzed in Section III. Section IV presents the details of designing a two-class OMCS-GMPLS network and the numerical results, including a comparison between the performance of the proposed OMCS-GMPLS network and that of the OCS-GMPLS network, which utilizes MLVW-OOCs. Finally, the paper is concluded in Section V.

\section{Multiservice OMCS-GMPLS NETWORK Architecture AND OPERATION}

In this section we describe the multiservice OMCSGMPLS network architecture and introduce a wavelength sharing code partitioning (WSCP) policy. In addition, we derive an expression of the blocking probability for each class of traffic.

\section{A. Network Architecture and Multicode Switching Mechanism}

In the proposed multiservice OMCS-GMPLS network, MCVW-OOC is utilized as the signature sequences. The MCVW-OOC family is characterized by $(L, w=$ $\left.\left\{w_{1}, w_{2}, \ldots, w_{Q}\right\}, D=\left\{d_{1}, d_{2}, \ldots, d_{Q}\right\}, F=\left\{F_{1}, F_{2}, \ldots, F_{Q}\right\}, Q, I\right)$, where $L$ is the fixed code length for all classes, $Q$ is the number of specified classes in the network, and $w_{j}, d_{j}$, and $F_{j}$ (for any $j \in \Omega=\{1,2, \ldots, Q\}$ ) denote the code weight, the fraction of codewords with weight $w_{j}$ in class $j$, and the number of parallel codes assigned to class $j$ users, respectively. In addition, $I$ indicates the cross-correlation matrix, which is defined as

$$
I \stackrel{\text { def }}{=}[I(n, m), \forall n, m \in \Omega],
$$

where $I(n, m)$ denotes the maximum cross correlation between the codewords in classes $n$ and $m$. It is clear that there are exactly $d_{i} \cdot|\mathcal{C}|$ codewords with weight $w_{j}$ in class $j$, where $|\mathcal{C}|$ represents the overall cardinality of available codewords per wavelength.

In a multiclass OMCS-GMPLS network, core labeled traffic is identified by the input/output port, wavelength, 
and optical code, $(i, \lambda, C)$, which are considered as the available network resources. Multicode data forwarding along a predefined path, called MCSP, is performed using wavelength switching, OC switching, or both. MCSP consists of a number of coded packets that follow the same path from source to destination. The number of codes in each MCSP is determined based on the required data rate for each forward-equivalent class (FEC). The label distribution protocol (LDP) determines the incoming and outgoing label of a path in each intermediate router and records this information in the label-lookup table of the corresponding intermediate routers. In order to provide multiclass MCSP, the label-lookup table is classified based on the data rate and QoS of each FEC. Moreover, LDP should be modified to assign simultaneously multiple labels based on each FEC required data rate. An edge optical multicode switching router (EOMCSR), which connects the OMCS-GMPLS core network to other networks, assigns a number of codes (labels) to the incoming packets based on their FECs and destination addresses. In the core network, the core optical multicode switching router (OMCSR) performs routing and forwarding functions by only recognizing the label $\left(i_{\text {in }}, \lambda_{\text {in }}, C_{\text {in }}\right)$ of the incoming optical data in the optical domain and determines the outgoing label $\left(i_{\text {out }}, \lambda_{\text {out }}, C_{\text {out }}\right)$ from its internal label-lookup table based on the incoming label.

Figure 1 shows a MC-based routing example in a simple multiservice OMCS-GMPLS network. It is assumed that there are two FECs, the high-bit rate class (FEC1), which assigns two codes simultaneously, and the low-bit rate class (FEC2), which assigns only one code. Moreover, four MCSPs are established, and their incoming/outgoing labels are recorded in the label-lookup table of the core OMCSRs. The number of labels assigned to each MCSP depends on its FEC. For instance, FEC1-MCSP1 assigns two labels and the packets are coded from EOMCSR1 to EOMCSR4 by $\left[\left(\lambda_{2}, C_{1}^{1}\right),\left(\lambda_{2}, C_{2}^{1}\right)\right],\left[\left(\lambda_{2}, C_{3}^{1}\right),\left(\lambda_{2}, C_{4}^{1}\right)\right]$, and $\left[\left(\lambda_{1}, C_{3}^{1}\right),\left(\lambda_{1}, C_{4}^{1}\right)\right]$. Based on the wavelength and code conversion of the incoming packets, four scenarios of label swapping can be used in MCSP; as shown in the OMCSR1 label-lookup table, FEC1MCSP1 and FEC2-MCSP4 packets are routed by changing the code and wavelength of the incoming packets, respectively, while FEC2-MCSP2 packets are forwarded without any conversion. From the OMCSR3 label-lookup table, FEC1-MCSP3 packets are forwarded by both wavelength and code conversion.

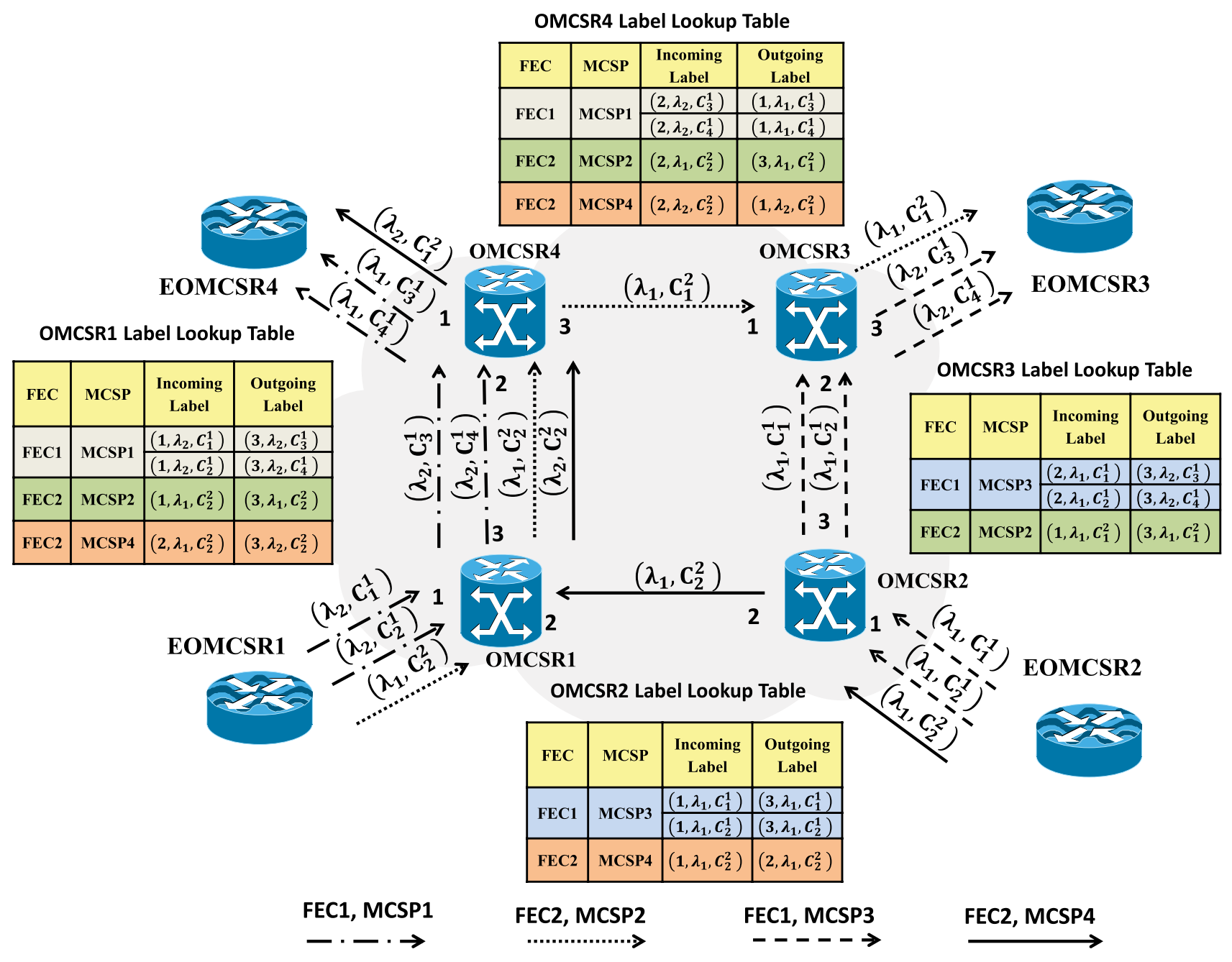

Fig. 1. Multiservice OMCS-GMPLS network architecture with end-to-end operation. 


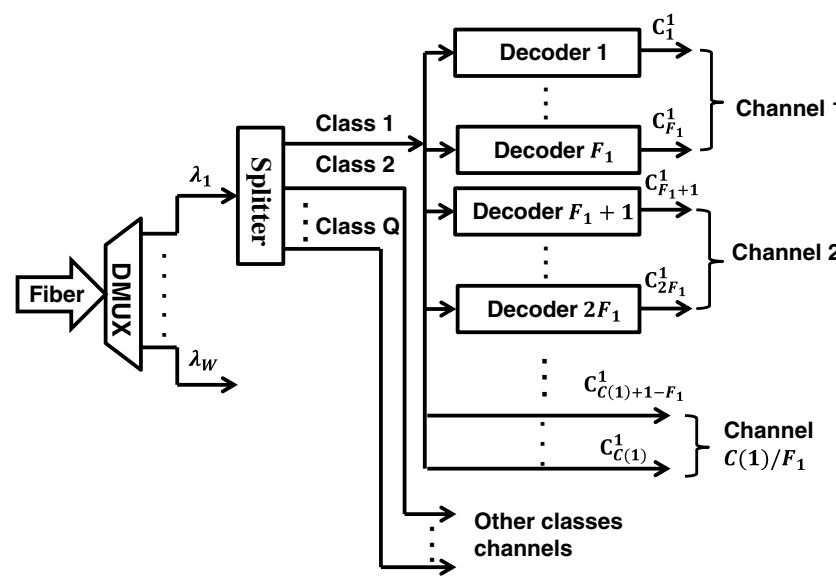

(a)

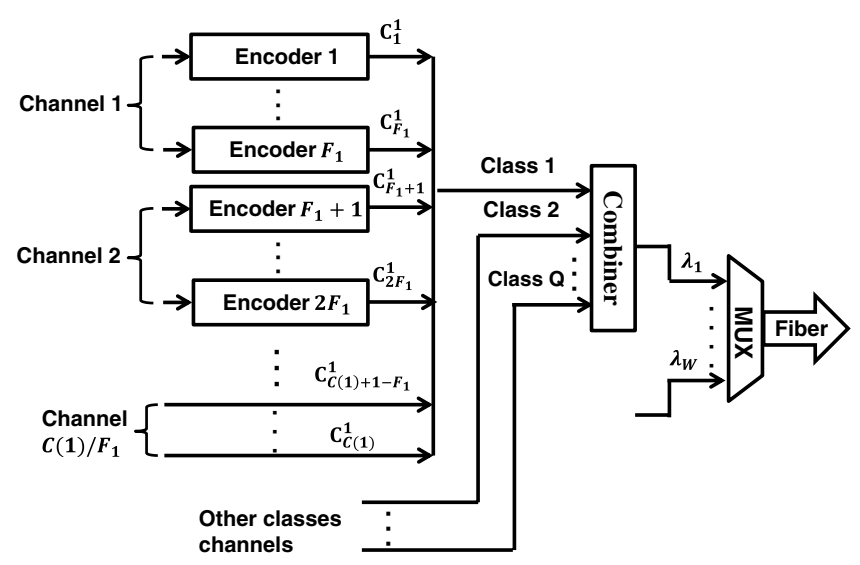

(b)

Fig. 2. Multiservice multicode decoder and encoder arrays used in OMCS-GMPLS router. (a) Multiservice multicode decoder array. (b) Multiservice multicode encoder array.

The architecture of OMCSR is similar to that of an optical code switching router (OCSR) presented in [23] with the difference that for class $j$, which requests $F_{j}$ codes, the controller allocates the required $F_{j}$ decoders and encoders simultaneously as shown in Fig. 2.

In Fig. 2, the wavelength demultiplexer first separates the received optical signal into $W$ components based on the wavelength. Every wavelength is split using an optical splitter through a $Q$ decoder array of the defined service classes, and in each decoder array $\mathcal{C}(j)$ different tapped delay line (TDL) decoders, each of which is matched to one of the $\mathcal{C}(j)$ designed codewords in class $j$. After decoding the incoming signals, the controller searches the label-lookup table to make a routing decision to determine the number of labels and which outgoing labels are assigned to the outgoing packets considering incoming label $\left(i_{\text {in }}, \lambda_{\text {in }}, C_{\text {in }}\right)$ and their FEC. If the incoming label wavelength $\lambda_{\text {in }}$ and outgoing label wavelength $\lambda_{\text {out }}$ are different, an optical cross-connect (OXC) first fed the output of the decoder into a tunable wavelength converter (TWC), which modulates it on a $\lambda_{\text {out }}$ selected by the controller and then feeds the output to the input of the corresponding encoder of $C_{\text {out }}$. After input label swapping, the outputs of the encoder arrays of different service classes are combined using an optical coupler, resulting in a new transmission signal in the wavelength. Then different wavelengths are multiplexed by the wavelength multiplexer, and forwarded to the next switching node.

\section{B. Wavelength Sharing Code Partitioning Policy}

Figure 3 illustrates the fiber bandwidth partitioning using the WSCP policy to provide $Q$ service classes in a OMCS-GMPLS network. We assume that the total number of wavelengths available in a single-fiber link is $W$, which are shared among all service classes. Isolation between different classes is performed by dividing the available codewords in each wavelength into $Q$ different code sets, each with $\mathcal{C}(j), j \in \Omega$ available codewords. The codes of each code set are assigned to one of the $Q$ classes according to the required QoS. In the WSCP policy, the performance and capacity of the network are limited by multiple access interference (MAI) from codes from the same service class and from other classes in the same wavelength.

The number of available codewords in class $j$ in each wavelength should achieve the following bound:

$$
0<\mathcal{C}(j)<|\mathcal{C}|, \quad j \in \Omega
$$

with the additional constraint on the overall cardinality $|\mathcal{C}|$ :

$$
|\mathcal{C}|=\sum_{j=1}^{Q} \mathcal{C}(j)=\sum_{j=1}^{Q} d_{j}|\mathcal{C}|
$$

In addition, we have the following constraint [22]:

$$
|\mathcal{C}| \leq \frac{(L-1)}{\sum_{j=1}^{Q} d_{j} w_{j}\left(w_{j}-1\right)} .
$$

It should be noticed that the number of available codes in class $j$ is

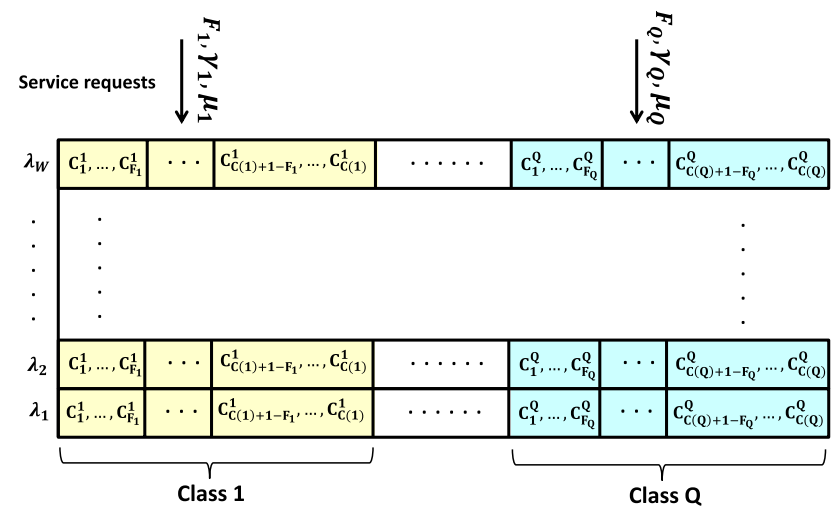

Fig. 3. Fiber bandwidth partitioning of OMCS-GMPLS network into $Q$ service classes using WSCP policy. 


$$
\mathcal{L}(j)=W \times \mathcal{C}(j)
$$

In Fig. 3, we assume that a connection request of each of the classes with $F_{j}, j \in \Omega$ simultaneous codewords (servers) requirements arrives according to a Poisson process with arrival rate $\gamma_{j}$ and exponentially distributed holding time with mean $1 / \mu_{j}$. Hence, class $j$ users are assigned simultaneous free $F_{j}$ codewords (i.e., $C_{1}^{j}, \ldots, C_{F_{j}}^{j}$ ) from the available code set in class $j$ in any wavelength.

\section{Blocking Probability}

Here, we are interested in the blocking probability of each class of traffic. A fiber link using the WSCP policy operates as $Q$ independent service classes. In this case, the OMCS-GMPLS link forms a multiservice loss system, where users of some classes may require more than one code (server) simultaneously. Notice that the $M / M / \mathcal{L}(j) / \mathcal{L}(j)$ Erlang's model is a multiserver singleservice loss model, while here we consider a multiserver multiservice loss model. The number of valid states for class $j$ traffic is $\Gamma(j)=\left\lfloor\mathcal{L}(j) / F_{j}\right\rfloor$, and the state transition diagram is as shown in Fig. 4. A transition from state $\left(n-F_{j}\right)$ to state $(n)$ is due to a new arrival of class $j$ request requiring any $F_{j}$ free codes with rate $\gamma_{j}$, while transition from state $(n)$ to state $\left(n-F_{j}\right)$ is due to a completion of class $j$ service with rate $F_{j} \mu_{j}$. Here $n$ denotes the number of occupied codes in class $j$. The unnormalized steady-state probability $\tilde{P}_{N}^{(j)}(n)$ of being in state $n$ can be calculated by Kaufman-Roberts recursion as follows [24,25]:

$$
\tilde{P}_{N}^{(j)}(n)= \begin{cases}0 ; & n<0, \\ 1 ; & n=0, \\ \frac{F_{j} A_{j}}{n} \tilde{P}_{N}^{(j)}\left(n-F_{j}\right) ; & 0<n<\mathcal{L}(j),\end{cases}
$$

where $A_{j}=\gamma_{j} / \mu_{j}$ denotes the offered traffic per idle user. The above probabilities are then normalized such that $\sum_{n=0}^{\mathcal{L}(j)} P_{N}^{(j)}(n)=1$ :

$$
P_{N}^{(j)}(n)=\frac{\tilde{P}_{N}^{(j)}(n)}{\sum_{k=0}^{\mathcal{L}(j)} \tilde{P}_{N}^{(j)}(k)} .
$$

When a connection request of service class $j$ arrives and cannot find $F_{j}$ free codes (servers), its service is denied and it is blocked and cleared from the system. Hence, the blocking probability $P_{B}^{(j)}$ for class $j$ can be found by adding up the steady-state probabilities of all states with code occupancies higher than $\mathcal{L}(j)-F_{j}$ :

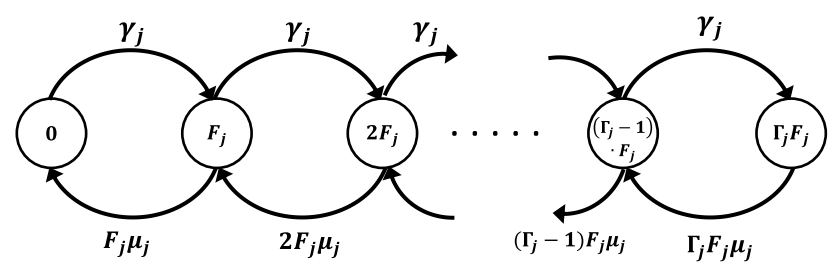

Fig. 4. State transition diagram for class $j$ with $F_{j}$ parallel codes.

$$
P_{B}^{(j)}=\sum_{n=\mathcal{L}(j)-F_{j}+1}^{\mathcal{L}(j)} P_{N}^{(j)}(n)
$$

The OMCS-GMPLS network can provide fine subwavelength granularity capacity to connections. Moreover, serving a class $j$ connection request that requires $F_{j}$ codewords is done by finding any $F_{j}$ unoccupied codewords in any wavelength, hence removing the spectrum contiguity constraints found in an OFDM-based elastic network [11]. However, the OMCS-GMPLS network poses some drawbacks; for example, serving class $j$ connection requests requires $F_{j}$ encoders/decoders in each OMCSR. In addition, high-rate classes will experience higher blocking probability than low-rate classes.

\section{Performance Evaluation}

In this section we derive expressions for the BER of the proposed network. Furthermore, we obtain expressions for both the probability of degradation and system throughput.

\section{A. Multiservice Multicode BER Evaluation}

In this subsection, the BER is derived for a multiservice MCVW-OOC based OMCS-GMPLS network for two receiver structures, namely, correlation receivers with and without hard limiters. In general, the performance of an OCDM-MCSP is primarily affected by MAI from other paths and from the codes in the same path. In this paper, we neglect the effects of shot noise, thermal noise, and other fiber impairments, and consider MAI as the main degrading factor to the system performance. This makes the OCDM-MCSP provisioning more straightforward. In addition, we assume a chip synchronous interference model, which results in an upper bound on the system performance [26]. We further assume that the network is power-controlled so that all packets arrive at the receiver with equal power; therefore all connections would create an equal amount of interference.

1) BER of a Correlation Receiver: In an $(L, w, D, F, Q, I)$ MCVW-OOC with correlation constraints equal to 1 , i.e., $[I(n, m)=1, \forall n, m \in \Omega]$, each interfering code may contribute only one chip interference with the intended user. Let $p_{k q}$ denote the probability that a codeword of class $q$ makes one chip interference with a codeword of class $k$. Since in a codeword of class $q, w_{q}$ marked chips out of $L$ chips may interfere with any of the $w_{k}$ marked chips of a class $k$ user, we can write

$$
p_{k q}=\frac{w_{k} w_{q}}{L}, \quad k, q \in \Omega .
$$

We assume that there are $K_{i}$ active users in class $i, i \in \Omega$. A user in class $i$ is assigned $F_{i}$ codewords, each of weight $w_{i}$. The total number of active codes in the network, $\mathcal{K}$, is thus

$$
\mathcal{K}=F_{1} K_{1}+F_{2} K_{2}+\cdots+F_{Q} K_{Q}
$$


Furthermore, there are

$$
\begin{aligned}
F_{1} K_{1} & +\cdots+F_{k-1} K_{k-1}+F_{k} K_{k}-1 \\
& +F_{k+1} K_{k+1}+\cdots+F_{Q} K_{Q}=\mathcal{K}-1
\end{aligned}
$$

codes that could interfere with the desired user in class $k$. The bit-error probability $P_{e(k)}$ of a class $k$ user, with $w_{k}$ code weight and $F_{k}$ parallel codes, given $\mathcal{K}$ codes in the network is evaluated as

$$
\begin{aligned}
P_{e(k)}= & \operatorname{Pr}\{\text { error } \mid \mathcal{K} \text { codes }\} \\
= & \sum_{\ell_{1}=0}^{F_{1} K_{1}} \ldots \sum_{\ell_{k}=0}^{F_{k} K_{k}-1} \cdots \sum_{\ell_{Q}=0}^{F_{Q} K_{Q}} \operatorname{Pr}\{\ell \text { interferers } \mid \mathcal{K} \text { codes }\} \\
& \times \operatorname{Pr}\{\text { a bit error } \mid \ell \text { interferers }, \mathcal{K} \text { codes }\},
\end{aligned}
$$

where we have defined the interfering vector

$$
\begin{aligned}
\ell \stackrel{\text { def }}{=}\left\{\left[\ell_{1}, \ell_{2}, \ldots, \ell_{Q}\right]: \ell_{j} \in\left\{0,1, \ldots, F_{j} K_{j}\right\}\right. \\
\quad \forall j \in\{1, \ldots k-1, k+1, \ldots, Q\}, \\
\left.\quad \ell_{k} \in\left\{0,1, \ldots, F_{k} K_{k}-1\right\}\right\} .
\end{aligned}
$$

The first conditional probability in the right-hand side of Eq. (12) can be evaluated as follows:

$$
\begin{aligned}
\operatorname{Pr}\{\ell \text { interferers } \mid \mathcal{K} \text { codes }\}= & \left(\begin{array}{c}
F_{k} K_{k}-1 \\
\ell_{k}
\end{array}\right) p_{k k}^{\ell_{k}}\left(1-p_{k k}\right)^{F_{k} K_{k}-1-\ell_{k}} \\
& \times \prod_{q=1 q \neq k}^{Q}\left(\begin{array}{c}
F_{q} K_{q} \\
\ell_{q}
\end{array}\right) p_{k q}^{\ell_{q}}\left(1-p_{k q}\right)^{F_{q} K_{q}-\ell_{q}},
\end{aligned}
$$

where $p_{k k}$ is given by Eq. (13) with $q=k$.

Assuming that data bits " 1 " and " 0 " are equally likely $(\operatorname{Pr}\{0\}=\operatorname{Pr}\{1\}=1 / 2)$, the last conditional probability in Eq. (12) is calculated (for a class $k$ user) as follows. The correlation receiver decides a data bit "1" was transmitted if the total received pulses $Z$ from all weighted chips is not less than a threshold Th. A data bit " 0 " is decided otherwise. For optimal operation, the decision threshold Th is set to code weight of the intended service class $w_{k}[\underline{26}]$. Thus,

\section{$\operatorname{Pr}\{$ a bit error $\mid \ell$ interferers, $\mathcal{K}$ codes $\}$}

$=\frac{1}{2} \operatorname{Pr}\{$ a bit error $\mid \ell$ interferers, $\mathcal{K}$ codes, 1 was sent $\}$

$+\frac{1}{2} \operatorname{Pr}\{$ a bit error $\mid \ell$ interferers, $\mathcal{K}$ codes, 0 was sent $\}$

$=\frac{1}{2} \operatorname{Pr}\left\{Z \geq w_{k} \mid \ell\right.$ interferers, $\mathcal{K}$ codes, 0 was sent $\}$

$=\frac{1}{2} \frac{1}{\prod_{i=1}^{Q} 2^{\ell_{i}}} \sum_{i_{1}, i_{2}, \ldots, i_{Q} \in \Psi} \prod_{i=1}^{Q}\left(\begin{array}{c}\ell_{i} \\ i_{i}\end{array}\right)$,

where

$$
\begin{aligned}
\Psi & \stackrel{\text { def }}{=}\left\{\left[i_{1}, i_{2}, \ldots, i_{Q}\right]: i_{j} \in\left\{0,1, \ldots, F_{j} K_{j}\right\}\right. \\
& \forall j \in\{1, \ldots k-1, k+1, \ldots, Q\}, i_{k} \in\left\{0,1, \ldots, F_{k} K_{k}-1\right\}, \\
& \left.w_{k} \leq \sum_{q=1}^{Q} i_{q} \leq \mathcal{K}-1\right\} .
\end{aligned}
$$

Finally, substituting in Eq. (12), we get

$$
\begin{aligned}
P_{e(k)}= & \sum_{\ell_{1}=0}^{F_{1} K_{1}} \ldots \sum_{\ell_{k}=0}^{F_{k} K_{k}-1} \ldots \sum_{\ell_{Q}=0}^{F_{Q} K_{Q}} \\
& \times\left(\begin{array}{c}
F_{k} K_{k}-1 \\
\ell_{k}
\end{array}\right) p_{k k}^{\ell_{k}}\left(1-p_{k k}\right)^{F_{k} K_{k}-1-\ell_{k}} \\
& \times \prod_{q=1 q \neq k}^{Q}\left(\begin{array}{c}
F_{q} K_{q} \\
\ell_{q}
\end{array}\right) p_{k q}^{\ell_{q}}\left(1-p_{k q}\right)^{F_{q} K_{q}-\ell_{q}} \\
& \times\left[\frac{1}{2} \frac{1}{\prod_{i=1}^{Q} 2^{\ell_{i}}} \sum_{i_{1}, i_{2}, \ldots, i_{Q} \in \Psi} \prod_{i=1}^{Q}\left(\begin{array}{c}
\ell_{i} \\
i_{i}
\end{array}\right)\right] .
\end{aligned}
$$

2) BER of a Correlation Receiver With a Hard Limiter: For the correlation receiver with a hard limiter, an error occurs only when a data bit " 0 " is transmitted while the number of interfering pulses in every weighted chip position of the codeword of intended service class $k$ is nonzero. We define

$$
\chi_{k} \stackrel{\text { def }}{=}\left\{1,2, \ldots, w_{k}\right\}
$$

Given that there are $\ell$ interfering users, each interfering at exactly one chip position, there is a set of possible interference patterns. To describe these patterns, we define a $Q \times$ $w_{k}$ interference matrix $\mathcal{L}$ whose element $\ell_{i}^{j}, i \in \Omega, j \in \chi_{k}$, represents the number of users (out of available $\ell_{i}$ class $i$ users) that interfere with the $j$ th weighted chip of the desired user:

$$
\mathcal{L} \stackrel{\text { def }}{=}\left(\begin{array}{cccc}
\ell_{1}^{1} & \ell_{1}^{2} & \ldots & \ell_{1}^{w_{k}} \\
\ell_{2}^{1} & \ell_{2}^{2} & \ldots & \ell_{2}^{w_{k}} \\
\ldots & & \ldots & \\
\ell_{Q}^{1} & \ell_{Q}^{2} & \ldots & \ell_{Q}^{w_{k}}
\end{array}\right) .
$$

Notice that the sum over the rows of $\mathcal{L}$ gives the vector $\ell$ as defined in Eq. (13). On the other hand, the sum over the columns of $\mathcal{L}$ gives the vector $\boldsymbol{\alpha}$, defined as

$$
\boldsymbol{\alpha}=\left\{\alpha_{1}, \alpha_{2}, \ldots, \alpha_{w_{k}}\right\}^{\text {def }}=\left\{\sum_{i=1}^{Q} \ell_{i}^{1}, \sum_{i=1}^{Q} \ell_{i}^{2}, \ldots, \sum_{i=1}^{Q} \ell_{i}^{w_{k}}\right\}
$$

Here $\alpha_{i}, i \in \chi_{k}$ represents the number of interfering users that overlap with the $i$ th pulse position of the class $k$ desired user. Since every interfering user contributes one and only one pulse, the interference matrix elements must satisfy 


$$
\begin{gathered}
\ell_{i}^{j} \geq 0, \quad \text { any } \quad i \in \Omega, \quad j \in \chi_{k}, \\
\sum_{i=1}^{Q} \sum_{j=1}^{w_{k}} \ell_{i}^{j}=\sum_{i=1}^{Q} \ell_{i}=\sum_{j=1}^{w_{k}} \alpha_{j}=\ell .
\end{gathered}
$$

For a given $\ell$ there is a set of matrices $\mathcal{F}_{\ell}$ that satisfy Eq. (21):

$$
\begin{gathered}
\mathcal{F}_{\ell} \stackrel{\text { def }}{=}\left\{\mathcal{L} \in \mathbb{N}^{Q \times w_{k}}: \sum_{i=1}^{Q} \sum_{j=1}^{w_{k}} \ell_{i}^{j}=\ell\right. \\
\text { and } \left.\ell_{i}^{j} \geq 0, \text { any } i \in \Omega, j \in \chi_{k}\right\},
\end{gathered}
$$

where $\mathbb{N}$ is the set of natural numbers. Based on the analyses in $[27,28]$, the bit-error probability $P_{e}(k)$ of a class $k$ user with $\bar{w}_{k}$ code weight and $F_{k}$ parallel codes given $\mathcal{K}$ codes in a multiservice multicode network can be evaluated as follows:

$$
\begin{aligned}
P_{e(k)}= & \operatorname{Pr}\{\text { error } \mid \mathcal{K} \text { codes }\} \\
= & \sum_{\ell_{1}=0}^{F_{1} K_{1}} \ldots \sum_{\ell_{k}=0}^{F_{k} K_{k}-1} \ldots \sum_{\ell_{Q}=0}^{F_{Q} K_{Q}} \operatorname{Pr}\{\ell \text { interferers } \mid \mathcal{K} \text { codes }\} \\
& \times \sum_{\mathcal{L} \in \mathcal{F}_{\ell}} P\left(\mathcal{L} ; \mathcal{F}_{\ell}\right) \cdot P_{b e(k)}(\mathcal{L}),
\end{aligned}
$$

where $P\left(\mathcal{L} ; \mathcal{F}_{\ell}\right)$ is the probability of occurrence of interference pattern $\mathcal{L} \in \mathcal{F}_{\ell}$ given $\ell=\left[\ell_{1}, \ell_{2}, \ldots, \ell_{Q}\right]$ interfering users, and $P_{b e(k)}(\mathcal{L})$ is the BER of a class $k$ user given interference pattern $\mathcal{L}$. A user is equally likely to interfere at any one of the $w_{k}$ pulse positions independent of all other users. Thus, pattern $\mathcal{L}$ can be produced in

$$
\frac{\ell !}{\sum_{i=1}^{Q} \ell_{i}^{1} ! \sum_{i=1}^{Q} \ell_{i}^{2} ! \ldots \sum_{i=1}^{Q} \ell_{i}^{w_{k}} !}=\frac{\ell !}{\alpha_{1} ! \alpha_{2} ! \ldots \alpha_{w_{k}} !}
$$

ways, each with probability $1 / w_{k}^{\ell}$. So $P\left(\mathcal{L} ; \mathcal{F}_{\ell}\right)$ is given by

$$
P\left(\mathcal{L} ; \mathcal{F}_{\ell}\right)=\frac{1}{w_{k}^{\ell}} \frac{\ell !}{\alpha_{1} ! \alpha_{2} ! \ldots \alpha_{w_{k}} !} .
$$

The bit-error probability $P_{b e(k)}(\mathcal{L})$ given interference pattern $\mathcal{L}$ is evaluated as follows:

$$
\begin{aligned}
P_{b e(k)}(\mathcal{L})= & \frac{1}{2} \operatorname{Pr}\{\text { a bit error } \mid \mathcal{L} \text { interferers, } 1 \text { was sent }\} \\
& +\frac{1}{2} \operatorname{Pr}\{\text { a bit error } \mid \mathcal{L} \text { interferers, } 0 \text { was sent }\} \\
= & \frac{1}{2} \operatorname{Pr}\left\{\alpha_{i} \geq 1, \quad \forall i \in \chi_{k} \mid \mathcal{L} \text { interferers, } 0 \text { was sent }\right\}
\end{aligned}
$$

The last equation can be rewritten as

$$
\begin{aligned}
& P_{b e(k)}(\mathcal{L}) \\
& =\frac{1}{2}-\frac{1}{2} \operatorname{Pr}\left\{\alpha_{i}=0, \text { some } i \in \chi_{k} \mid \mathcal{L} \text { interferers, 0 was sent }\right\} \\
& \quad=\frac{1}{2}-\frac{1}{2}\left(\sum_{m=1}^{w_{k}} \frac{1}{2^{\alpha_{m}}}-\sum_{m=1}^{w_{k}-1} \sum_{n=m+1}^{w_{k}} \frac{1}{2^{\alpha_{m}+\alpha_{n}}}+\cdots+(-1)^{w_{k}-1} \frac{1}{2^{\ell}}\right) .
\end{aligned}
$$

In the above relation, we have used the inclusion-exclusion property of the probability of a union of events. Substituting in Eq. (23), we obtain the BER of a class $k$ user $P_{e(k)}$ for a correlation receiver with a hard limiter as

$$
\begin{aligned}
P_{e(k)}= & \sum_{\ell_{1}=0}^{F_{1} K_{1}} \ldots \sum_{\ell_{k}=0}^{F_{k} K_{k}-1} \cdots \sum_{\ell_{Q}=0}^{F_{Q} K_{Q}} \\
& \times\left(\begin{array}{c}
F_{k} K_{k}-1 \\
\ell_{k}
\end{array}\right) p_{k k}^{\ell_{k}}\left(1-p_{k k}\right)^{F_{k} K_{k}-1-\ell_{k}} \\
& \times \prod_{q=1 q \neq k}^{Q}\left(\begin{array}{c}
F_{q} K_{q} \\
\ell_{q}
\end{array}\right) p_{k q}^{\ell_{q}}\left(1-p_{k q}\right)^{F_{q} K_{q}-\ell_{q}} \\
& \times \sum_{\mathcal{L} \in \mathcal{F}_{\ell}: \alpha_{i} \geq 1 \forall i \in \chi_{k}} \frac{1}{w_{k}^{\ell}} \frac{\ell !}{\alpha_{1} ! \alpha_{2} ! \ldots \alpha_{w_{k}} !} \\
& \times\left[\frac{1}{2}-\frac{1}{2}\left(\sum_{m=1}^{w_{k}} \frac{1}{2^{\alpha_{m}}}-\sum_{m=1}^{w_{k}-1} \sum_{n=m+1}^{w_{k}} \frac{1}{2^{\alpha_{m}+\alpha_{n}}}\right.\right. \\
& \left.\left.+\cdots+(-1)^{w_{k}-1} \frac{1}{2^{\ell}}\right)\right] .
\end{aligned}
$$

\section{B. Probability of Degradation}

The OMCS-GMPLS network must guarantee the QoS requested by each user, and at the same time maximize the network capacity by maximizing the number of users admitted to the network. In an OMCS-GMPLS network, the performance is limited by MAI and the BER is a function of the number of simultaneously active MCSPs. Consequently, the allowable number of simultaneous MCSPs must be controlled according to the maximum BER for each class; otherwise, the desired QoS would be drastically affected. Capacity can be improved significantly by allowing graceful QoS degradation. In this paper, we present a call admission control (CAC) protocol that depends on degrading the desired QoS (in terms of BER) of class $j$ active users by increasing the number of admitted users over the degradation threshold $\Gamma_{\mathrm{Th}}^{(j)}$ of class $j$, which represents the maximum number of users (each with $F_{j}$ parallel codes) that may be simultaneously active in each wavelength on the network for a given BER threshold.

If $\rho$ represents the probability that a connected MCSP transmits data, then the probability density function (PDF) of the number of simultaneously active codes $x$ in class $j$ in a wavelength is given by [23]

$$
\begin{aligned}
P_{x}^{(j)}(x)= & \sum_{m=x}^{\mathcal{C}(j)} P(x \text { active paths } \mid m \text { connected paths }) \\
& \times P_{M}^{(j)}(m \text { connected paths }) \\
= & \sum_{m=x}^{\mathcal{C}(j)}\left(\begin{array}{c}
m \\
x
\end{array}\right) \rho^{x}(1-\rho)^{m-x} P_{M}^{(j)}(m)
\end{aligned}
$$

where $P_{M}^{(j)}(\cdot)$ denotes the PDF of the number of occupied codes per wavelength in class $j$. It can be evaluated as follows. When a connection request arrives, a wavelength is randomly chosen and $F_{j}$ of its unused codes is assigned 
simultaneously to the arrived service-class $j$ connection request. Assuming that $\mathcal{C}(j)$ is a multiple of $F_{j}$, we get the conditional PDF of the number of occupied codes in a given wavelength by modifying the hypergeometric distribution to account for simultaneously assigning $F_{j}$ codes from the same wavelength to each incoming connection request as follows:

$$
P_{M}^{(j)}(m \mid n)=\frac{\left(\begin{array}{c}
\mathcal{C}(j) \\
m
\end{array}\right) \sum_{r \in \mathcal{R}} \prod_{i=1}^{W-1}\left(\begin{array}{c}
\mathcal{C}(j) \\
r_{i} F_{j}
\end{array}\right)}{\sum_{n \in \mathcal{N}} \prod_{i=1}^{W}\left(\begin{array}{c}
\mathcal{C}(j) \\
n_{i} F_{j}
\end{array}\right)},
$$

where $n$ and $m$ are the number of occupied codes in a fiber and a wavelength, respectively, and the two sets $\mathcal{N}$ and $\mathcal{R}$ are defined as

$$
\begin{gathered}
\mathcal{N} \stackrel{\text { def }}{=}\left\{\boldsymbol{n}=\left[n_{1}, n_{2}, \ldots, n_{W}\right]: \sum_{i=1}^{W} n_{i}=\frac{n}{F_{j}} \quad\right. \text { and } \\
\left.n_{i} \in\left\{0,1, \ldots, \frac{\mathcal{C}(j)}{F_{j}}\right\} \quad \forall i \in\{1,2, \ldots, W\}\right\}, \\
\mathcal{R} \stackrel{\text { def }}{=}\left\{r=\left[r_{1}, r_{2}, \ldots, r_{W-1}\right]: \sum_{i=1}^{W-1} r_{i}=\frac{n-m}{F_{j}}\right. \text { and } \\
\left.r_{i} \in\left\{0,1, \ldots, \frac{\mathcal{C}(j)}{F_{j}}\right\} \quad \forall i \in\{1,2, \ldots, W-1\}\right\} .
\end{gathered}
$$

Using the PDF of $n$, we have

$$
P_{M}^{(j)}(m)=\sum_{n=m}^{\mathcal{L}(j)} \frac{\left(\begin{array}{c}
\mathcal{C}(j) \\
m
\end{array}\right) \sum_{\forall k_{i} \mathrm{sets}} \prod_{i=1}^{W-1}\left(\begin{array}{c}
\mathcal{C}(j) \\
k_{i} F_{j}
\end{array}\right)}{\sum_{n_{i} \mathrm{sets}} \prod_{i=1}^{W}\left(\begin{array}{c}
\mathcal{C}(j) \\
n_{i} F_{j}
\end{array}\right)} P_{N}^{(j)}(n) .
$$

In the WSCP policy, if at least the number of active codes in one service class exceeds $F_{j} \Gamma_{\mathrm{Th}}^{(j)}$, the desired QoS is degraded. In this case, $P_{\text {deg }}^{(j)}$ is the same for all classes and (after dropping the index $j$ ) is given as

$$
\begin{aligned}
P_{\mathrm{deg}} & =\operatorname{Pr}\left\{x^{(j)}>F_{j} \Gamma_{\mathrm{Th}}^{(j)}, \text { some } j \in \Omega\right\} \\
& =1-\operatorname{Pr}\left\{x^{(j)} \leq F_{j} \Gamma_{\mathrm{Th}}^{(j)}, \quad \forall j \in \Omega\right\} .
\end{aligned}
$$

Considering the statistical independence among the different classes, the total probability of degradation is obtained by using the probability of degradation of each class as follows:

$$
P_{\mathrm{deg}}=1-\prod_{j=1}^{Q}\left[P_{x}^{(j)}\left(x^{(j)} \leq F_{j} \Gamma_{\mathrm{Th}}^{(j)}\right)\right],
$$

where $P_{x}^{(j)}(\cdot)$ denotes the probability of getting the required QoS for class $j$. Using Eq. (29) and assuming that $\rho$ is the same for all classes, we can write

$$
\begin{aligned}
P_{x}^{(j)} & \left(x^{(j)} \leq F_{j} \Gamma_{\mathrm{Th}}^{(j)}\right) \\
= & \sum_{x^{(j)}=0}^{F_{j} \Gamma_{\mathrm{Th}}^{(j)}}\left[\sum_{m=x^{(j)}}^{\mathcal{C}(j)}\left(\begin{array}{c}
m \\
x^{(j)}
\end{array}\right) \rho^{x(j)}(1-\rho)^{m-x(j)} P_{M}^{(j)}(m)\right] .
\end{aligned}
$$

Hence, $P_{\mathrm{deg}}$ can be computed as follows:

$$
\begin{aligned}
P_{\mathrm{deg}}= & 1-\prod_{j=1}^{Q}\left(\sum _ { x ^ { ( j ) } = 0 } ^ { F _ { j } \Gamma _ { \mathrm { Th } } ^ { ( j ) } } \left[\sum_{m=x^{(j)}}^{\mathcal{c}(j)}\left(\begin{array}{c}
m \\
x^{(j)}
\end{array}\right)\right.\right. \\
& \left.\left.\times \rho^{x(j)}(1-\rho)^{m-x(j)} P_{M}^{(j)}(m)\right]\right) .
\end{aligned}
$$

\section{Call Admission Control}

With CAC, the network blocks some new connection requests in order to reduce interference on the network so that the degradation probability decreases. However, this improvement in service availability comes at the cost of increasing the blocking probability.

For an OCDM-based OMCS-GMPLS network to be operated with perfect service availability, so that $P_{\mathrm{deg}}=0$ for any load, it follows that no more than $\Gamma_{\text {Th }}$ connected MCSPs can ever be connected to the network. However, this scheme can severely limit the number of available codes, since it eliminates the capacity gained through statistical multiplexing. On the other hand, the capacity of the network can be greatly increased if degradations are allowed to occur with some small probability. Define $\Lambda_{\text {Th }}$ as a blocking threshold that determines the upper bound of the number of active codes per wavelength. The value of $\Lambda_{\mathrm{Th}}$ is determined based on the activity coefficient such that $P_{\text {deg }}$ does not exceed a given degradation constraint $P_{\mathrm{deg}}^{\max }$, defined by the network operator. In other words, new connection requests for class $j$ are blocked when active codes $x^{(j)}>W \times \Lambda_{\mathrm{Th}}^{(j)}-F_{j}$, and as before, degradation occurs when $x^{(j)}>F_{j} \Gamma_{\mathrm{Th}}^{(j)}$. Thus, the blocking and degradation probabilities for class $j$ are given by

$$
\begin{aligned}
P_{B}^{(j)}= & \sum_{x^{(j)}=W \times \Lambda_{\mathrm{Th}}^{(j)}-F_{j}+1}^{W \times \Lambda_{\mathrm{Th}}^{(j)}} P_{N}^{(j)}\left(x^{(j)}\right), \\
P_{\mathrm{deg}}= & 1-\prod_{j=1}^{Q}\left(\sum _ { x ^ { ( j ) } = 0 } ^ { F _ { j } \Gamma _ { \mathrm { Th } } ^ { ( j ) } } \left[\sum_{m=x^{(j)}}^{\Lambda_{\mathrm{Th}}^{(j)}}\left(\begin{array}{c}
m \\
x^{(j)}
\end{array}\right)\right.\right. \\
& \left.\left.\times \rho^{x(j)}(1-\rho)^{m-x(j)} P_{M}^{(j)}(m)\right]\right),
\end{aligned}
$$

respectively. It should be noticed that the degradation becomes more probable and blocking becomes less probable as the blocking threshold $\Lambda_{\mathrm{Th}}^{(j)}$ increases. That is, there is a trade-off between the degradation and blocking properties of the system.

\section{System Throughput}

We consider a synchronous random access system, where packet transmissions start at the beginning of time slots, each of duration $T$. All packets are assumed to have a fixed length of $L_{B}$ bits, which corresponds to the slot 
duration $T$. When a class $j$ user becomes active, it is assigned $F_{j}$ codewords as long as the total number of occupied codes is less than the maximum number of available codewords. The active class $j$ user starts $F_{j}$ simultaneous packets transmission (with probability $p_{o}$ ) and enters a thinking mode. For unsuccessfully received packets, the user enters a backlogged mode and attempts to retransmit these packets after a random time delay with average $d$ time slots (with probability $p_{r}=1 / d$ ) [28]. A data packet is successfully received at the destination node if it passes through all intermediate core nodes without blocking and all its bits are received without errors.

Let $\ell_{k}, k \in \Omega$ denote the number of class $k$ transmitting users. Each user has $F_{k}$ simultaneous packets per time slot. Assuming that $p_{o}=p_{r}$ and $\ell_{k}$ is large enough, the composite arrival (new and retransmitted packets) distribution of a class $k$ packet is Poisson with a finite arrival rate $\gamma_{k}$ packets/s. The steady-state probability distribution of class $k$ composite arrivals is [29]

$$
f\left(\ell_{k}\right)=\frac{\left(G_{k} / F_{k}\right)^{\ell_{k}}}{\ell_{k} !} \exp \left(-G_{k} / F_{k}\right),
$$

where $G_{k}=\gamma_{k} T$ denotes the offered load of class $k$ packets, which is the average number of generated packets from class $k$ in one slot duration. The per node class $k$ steadystate throughput is thus

$$
\begin{aligned}
\beta_{k}= & \sum_{i=1, i \neq k}^{Q} \sum_{\ell_{i}=0}^{\infty} \sum_{\ell_{k}=0}^{\infty}\left[\ell_{k} F_{k} \wedge W \mathcal{C}(k)\right] \\
& \times P_{\operatorname{Succ}(k)} \prod_{i=1}^{Q} \frac{\left(G_{i} / F_{i}\right)^{\ell_{i}}}{\ell_{i} !} \exp \left(-G_{i} / F_{i}\right),
\end{aligned}
$$

where $x \wedge y$ denotes the minimum of two numbers $x$ and $y$, and $P_{\text {Succ }(k)}$ is the overall packet success probability of class $k$ packet transmission, given by

$$
P_{\text {Succ }(k)}=\left(1-P_{B}^{(k)}\right) P_{S(k)}\left(\left\lceil\frac{H}{W}\right\rceil\right),
$$

where $H=\ell_{1} F_{1} \wedge W \mathcal{C}(1)+\cdots+\ell_{Q} F_{Q} \wedge W \mathcal{C}(Q), \quad\lceil\cdot\rceil$ denotes the ceiling function, and $P_{S(k)}(\mathcal{K})$ represents the packet success probability (the probability that all of the bits of the class $k$ packet are correctly received at the destination) given $\mathcal{K}$ codes. For the correlation receiver, $P_{S(k)}(\mathcal{K})$ is given as

$$
\begin{aligned}
P_{S(k)}(\mathcal{K})= & \sum_{\ell_{1}=0}^{F_{1} K_{1}} \ldots \sum_{\ell_{k}=0}^{F_{k} K_{k}-1} \ldots \sum_{\ell_{Q}=0}^{F_{Q} K_{Q}} \\
& \times P(\ell \text { interferers } \mid \mathcal{K} \operatorname{codes})\left[P_{b c(k)}(\ell)\right]^{L_{B},}
\end{aligned}
$$

where $P(\ell$ interferers $\mid \mathcal{K}$ codes $)$ is given in Eq. (14) and the conditional bit-correct probability $P_{b c(k)}(\ell)$ is given as

$$
\begin{aligned}
P_{b c(k)}(\ell) & =P_{b c(k)}(\text { a bit success } \mid \ell \text { interferers }, \mathcal{K} \text { codes }) \\
& =1-P_{b e(k)}(\text { a bit error } \mid \ell \text { interferers, } \mathcal{K} \text { codes }) \\
& =1-\frac{1}{2} \frac{1}{\prod_{i=1}^{Q} 2^{\ell_{i}}} \sum_{i_{1}, i_{2}, \ldots, i_{Q} \in \Psi} \prod_{i=1}^{Q}\left(\begin{array}{c}
\ell_{i} \\
i_{i}
\end{array}\right) .
\end{aligned}
$$

For the correlation receiver with a hard limiter, on the other hand, the packet success probability is given as

$$
\begin{aligned}
P_{S(k)}(\mathcal{K})= & \sum_{\ell_{1}=0}^{F_{1} K_{1}} \ldots \sum_{\ell_{k}=0}^{F_{k} K_{k}-1} \ldots \sum_{\ell_{Q}=0}^{F_{Q} K_{Q}} \\
\times & P(\ell \text { interferers } \mid \mathcal{K} \text { codes }) \\
& \times \sum_{\mathcal{L} \in \mathcal{F}_{\ell}} P\left(\mathcal{L} ; \mathcal{F}_{\ell}\right)\left[P_{b c(k)}(\mathcal{L})\right]^{L_{B}},
\end{aligned}
$$

where the conditional bit-correct probability $P_{b c(k)}(\mathcal{L})$ is given as

$$
P_{b c(k)}(\mathcal{L})=1-P_{b e(k)}(\mathcal{L})
$$

and $P_{b e(k)}(\mathcal{L})$ is given by Eq. $\underline{(27)}$.

\section{NumericAl Results}

In this section we investigate the performance of the OMCS-GMPLS network. Considering only two levels, namely, high and low levels, for data rate and QoS performance metrics, there are $2^{2}$ possible service classes. For the sake of simplicity, we will consider only two service classes defined as follows:

1) Class 1: High rate and high QoS $\left(B E R \leq 10^{-9}\right)$,

2) Class 2: Low rate and low $\mathrm{QoS}\left(\mathrm{BER} \leq 10^{-6}\right)$,

where class 1 is suitable for real-time video streaming and class 2 is appropriate for real-time voice transmissions.

For the sake of comparison, we use the previously proposed MLVW-OOC technique [23] for providing multirate multiservice in the OCS-GMPLS network. For this network, the following parameters are adopted: $L=$ $\{600,1200\}, \quad w=\{7,5\}, \quad D=\{14 / 42,28 / 42\}, \quad F=\{1,1\}$, and $Q=2, I=1$. In order to provide the same transmission rate and QoS using our proposed MCVW-OOC technique, the following parameters are adopted: $L=$ $\{1200,1200\}, w=\{7,5\}, D=\{14 / 42,28 / 42\}, F=\{2,1\}$, and $Q=2, I=1$. Taking $W=4$, the number of the available codewords in classes 1 and 2 are $\mathcal{L}(1)=W \times \mathcal{C}(1)=56$ and $\mathcal{L}(2)=W \times \mathcal{C}(2)=112$, respectively.

\section{A. Bit-Error Rate}

The BER performance of the two-class system using MLVW-OOC and MCVW-OOC is plotted in Fig. 5(a) as a function of the number of class 1 (high-QoS) active codes. The number of class 2 active codes is fixed to 10 and 14 for correlation receivers without and with a hard limiter, respectively, for both MLVW-OOC and MCVW-OOC systems. In Fig. 5(b), the BER is plotted versus the number of class 2 (low-QoS) active codes. For the case of the MLVW-OOC system, the number of class 1 active users is fixed to 4 and 8 with $F_{1}=1$ for correlation receivers without and with a hard limiter, respectively. For the case of the 


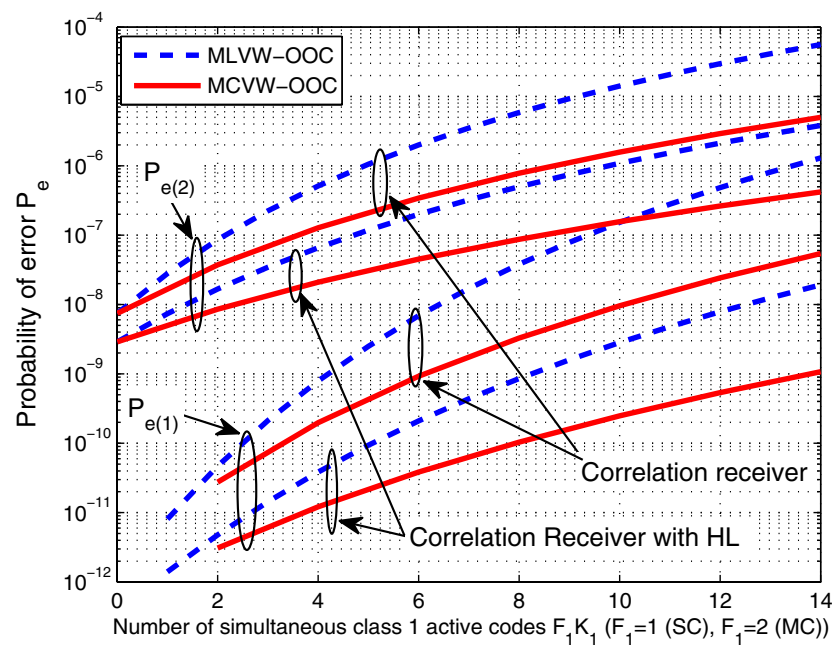

(a)

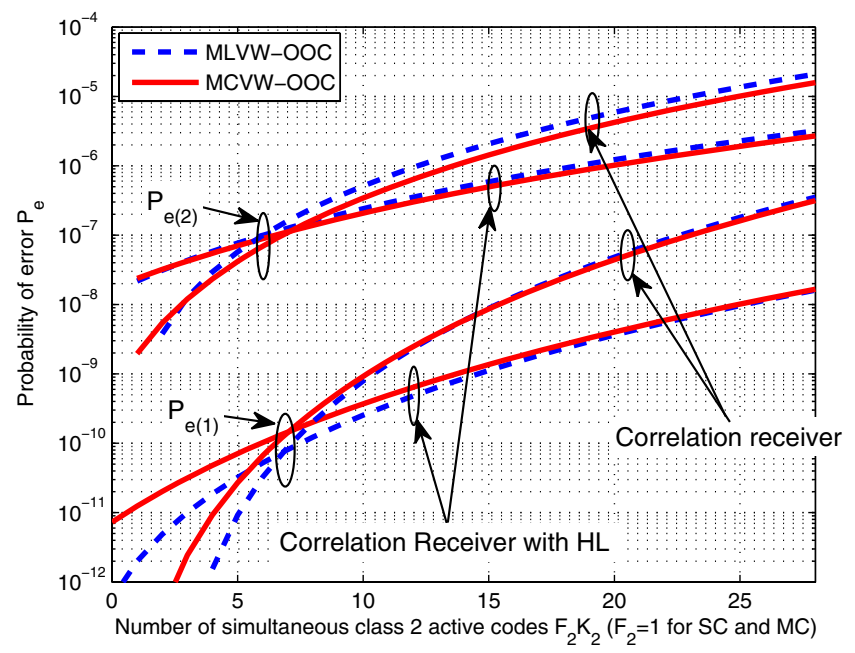

(b)

Fig. 5. BER versus number of simultaneous active codes for MLVW-OOC and MCVW-OOC systems.

MCVW-OOC system, however, the number of class 1 users is fixed to 3 and 7 with $F_{1}=2$ for correlation receivers without and with a hard limiter, respectively. It can be seen from the figure that the proposed MCVW-OOC system outperforms MLVW-OOC in terms of BER. Indeed, in order to meet the BER requirement $\left(\leq 10^{-9}\right.$ for class 1 users and $\leq 10^{-6}$ for class 2 users), the degradation threshold of class 1 and class 2 cannot exceed $\Gamma_{\mathrm{Th}}^{(1)}=4$ and $\Gamma_{\mathrm{Th}}^{(2)}=10$ for the correlation receiver, and $\Gamma_{\mathrm{Th}}^{(1)}=8$ and $\Gamma_{\mathrm{Th}}^{(2)}=14$ for the correlation receiver with the hard limiter in the case of the MLVW-OOC system. On the other hand, for the case of the MCVW-OOC system, in order to meet the same BER requirements, the degradation threshold of class 1 and class 2 cannot exceed $\Gamma_{\mathrm{Th}}^{(1)}=3(6$ codes $)$ and $\Gamma_{\mathrm{Th}}^{(2)}=10$ for the correlation receiver, and $\Gamma_{\mathrm{Th}}^{(1)}=7$ (14 codes) and
$\Gamma_{\mathrm{Th}}^{(2)}=14$ for the correlation receiver with a hard limiter. That is, it is clear that the number of supported highQoS users is almost the same for both MCVW-OCC and MLVW-OOC systems using a correlation receiver with a hard limiter. In contrast, the number of available codes in the MCVW-OOC system that meet the BER requirements for each class is larger than that in the MLVWOOC system. This is due to decreased interference between different classes in the MCVW-OOC system.

\section{B. Probability of Degradation}

The $P_{\text {deg }}$ of both OCS-GMPLS and OMCS-GMPLS networks, using correlation receivers with and without a hard limiter, is plotted in Fig. 6 versus the offered load for

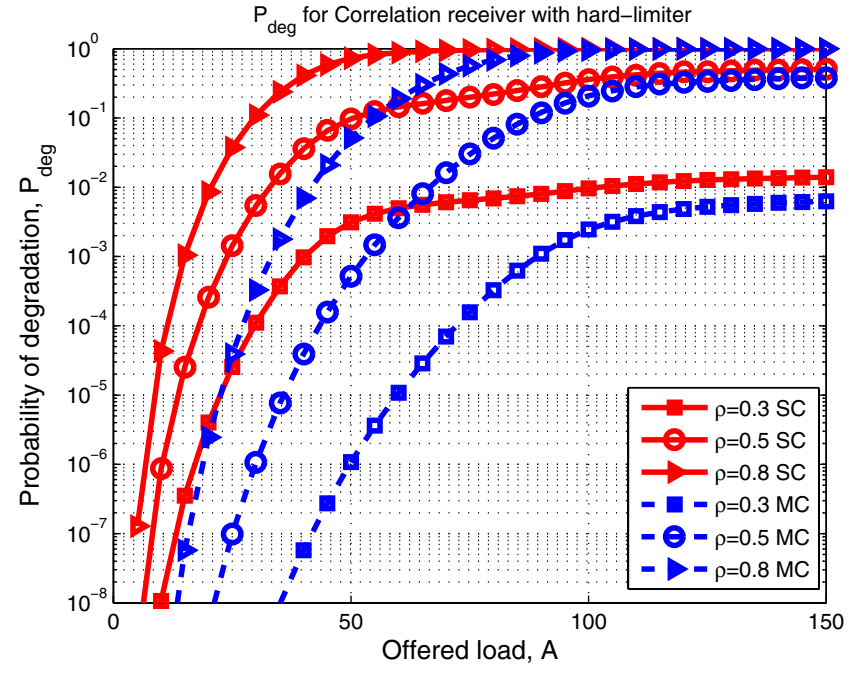

(a)

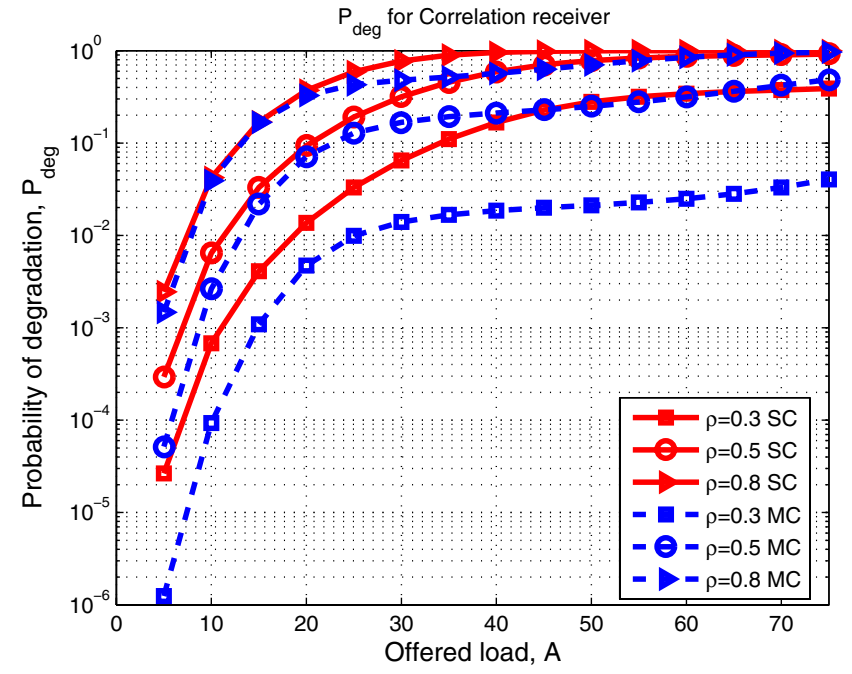

(b)

Fig. 6. $\quad P_{\text {deg }}$ as a function of offered load $A$ for different activity coefficients $\rho$. (a) Correlation receiver with hard limiter. (b) Correlation receiver. 
different activity coefficients. In these plots we consider the values of $\Gamma_{\text {th }}^{(i)}$ obtained from Fig. 5. Since the OCDMA-based GMPLS network is interference limited, when transmission is very bursty $(\rho \rightarrow 0)$, the total interference on the network decreases, causing the $P_{\text {deg }}$ to decrease and the number of available codes to increase. When the transmission is less bursty $(\rho \rightarrow 1)$, however, the $P_{\text {deg }}$ increases owing to the increase of the number of simultaneously active paths. Moreover, as the offered load increases, the number of connected paths increases, causing the degradation $P_{\text {deg }}$ to increase. Comparing the two receiver models, one can observe the superiority of the correlation receiver with a hard limiter. This is owing to the fact that the number of available codes when using the correlation receiver with a hard limiter is more than that when using the correlation receiver without a hard limiter. Furthermore, the

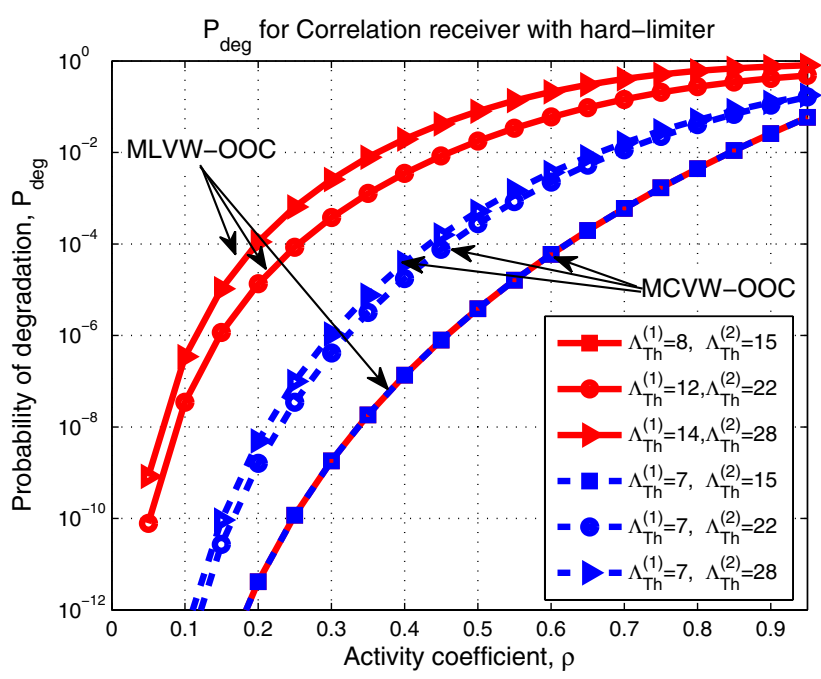

Fig. 7. Effect of limiting the number of connected paths on the performance.

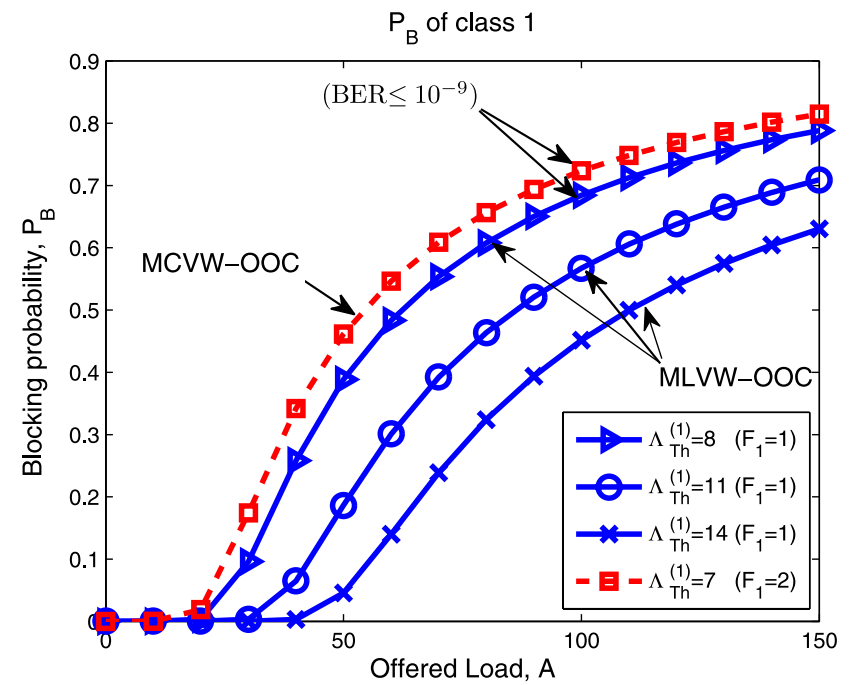

(a) results show the superiority of the OMCS-GMPLS network. This is due to the fact that the available codes in OMCS-GMPLS systems are more than those in OCS-GMPLS systems due to reduced interference, which results in increasing the number of available codes and reducing $P_{\text {deg }}$.

$P_{\text {deg }}$ is plotted in Fig. 7 versus the activity coefficient $\rho$ at an offered load $A=50$ for both MLVW-OOC and MCVWOOC systems. This indicates how the performance can be improved by limiting the number of connected paths. It can be seen from the figure that a decrease in blocking threshold $\Lambda_{\text {Th }}$ leads to an improvement in performance. The results show that the MCVW-OOC system outperforms the MLVW-OOC system since it can support more codes for the same QoS. As an example, for the case of the lowest possible blocking threshold, $P_{\text {deg }}$ is the same for both systems. However, the MLVW-OOC system can support 8 and 15 codes in class 1 (high-QoS) and class 2 (low-QoS), respectively, while the MCVW-OOC system can support $(7 \times 2=14)$ and 15 codes in class 1 and class 2 , respectively. For the case of the largest possible blocking threshold, both systems support the same number of codes (14 and 28 for class 1 and class 2, respectively); however, the performance of the MCVW-OOC system is better due to the reduced interference. Moreover, for the MCVWOOC system, the class 1 blocking threshold equals the degradation threshold $\left(\Lambda_{\mathrm{Th}}^{(1)}=\Gamma_{\mathrm{Th}}^{(1)}\right)$, and, hence, the QoS degradation occurs only if the number of active connected MCSPs in class 2 exceeds its corresponding degradation threshold $\Lambda_{\mathrm{Th}}^{(2)}>\Gamma_{\mathrm{Th}}^{(2)}$.

\section{Blocking Probability}

The blocking probabilities $P_{B}$ of class 1 and class 2 users are plotted in Fig. 8 versus the offered load $A$ for both MLVW-OOC and MCVW-OOC systems under different blocking thresholds $\Lambda_{\mathrm{Th}}$. As can be seen from the figure,

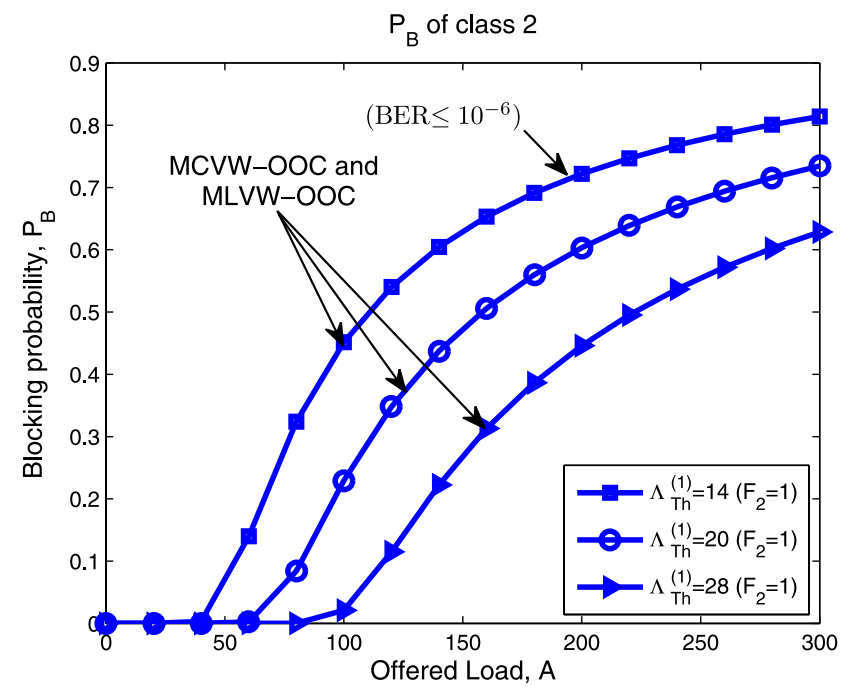

(b)

Fig. 8. Blocking probability versus offered load for different blocking thresholds. (a) For class 1 users. (b) For class 2 users. 


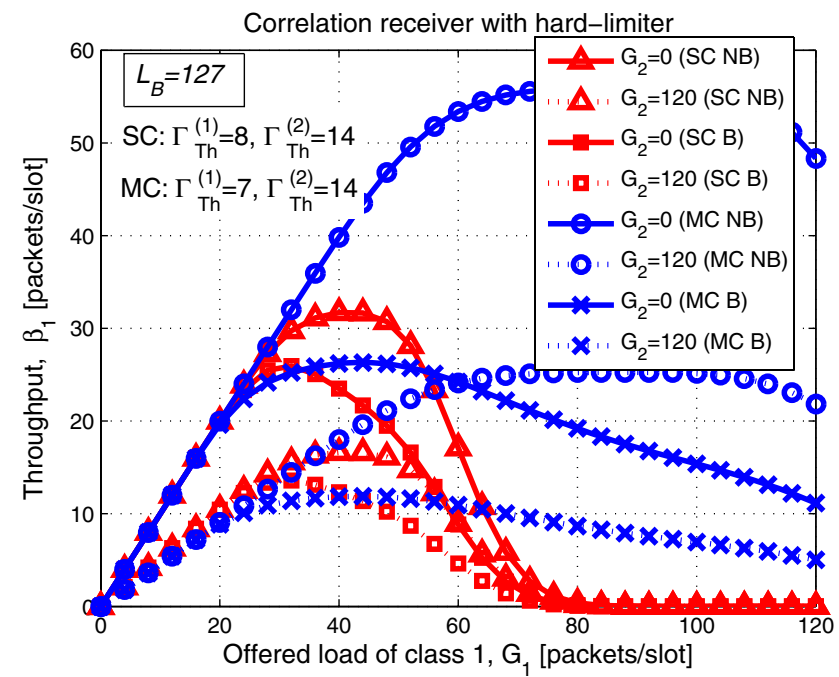

(a)

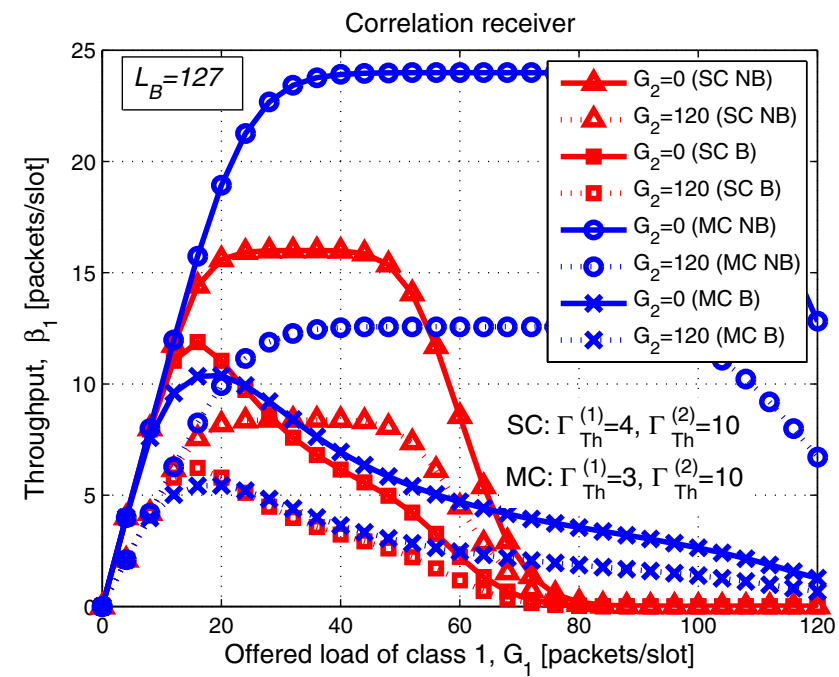

(b)

Fig. 9. Class 1 throughput versus offered packet load. (a) Correlation receiver with hard limiter. (b) Correlation receiver.

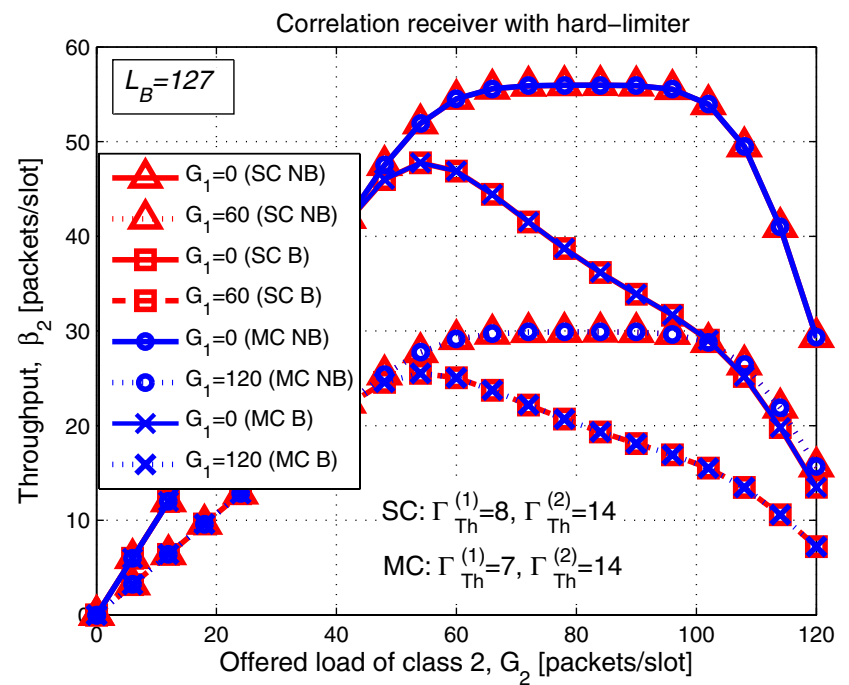

(a)

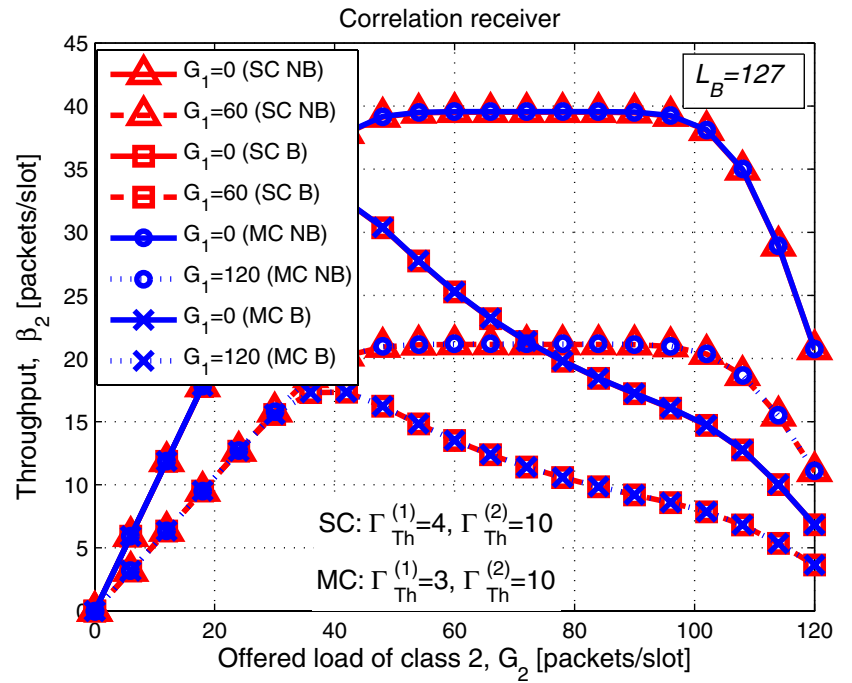

(b)

Fig. 10. Class 2 throughput versus offered packet load. (a) Correlation receiver with hard limiter. (b) Correlation receiver.

higher offered loads can be supported for class 2 users rather than class 1 users. This is clear as class 2 users have more available codes. Furthermore, $P_{B}$ increases as $\Lambda_{\mathrm{Th}}^{(i)}$ decreases. This is because the decrease in $\Lambda_{\mathrm{Th}}^{(i)}$ would reduce the number of available codes. In addition, it is clear from the figure that for class 1 users at BER $\leq 10^{-9}$, the MCVW-OOC system with $\Lambda_{\mathrm{Th}}^{(1)}=7(7 \times 2=14)$ experiences a higher blocking probability than that of the MLVW-OOC system with $\Lambda_{\mathrm{Th}}^{(1)}=8$. This is because the blocking in the MCVW-OOC system occurs when the connection request finds less than two free codes, while it occurs in the MLVW-OOC system when there is no free code. On the other hand, for class 2 users, the blocking probability is the same for both MCVW-OOC and MLVW-OOC systems since class 2 users in both systems require only one code.

\section{System Throughput}

The steady-state throughputs of both classes 1 and 2 are plotted as functions of the offered packet load in Figs. 9 and 10, respectively. Both MLVW-OOC and MCVW-OOC systems, using correlation receivers with and without a hard limiter, are adopted. In order to meet the BER requirement ( $\leq 10^{-9}$ for class 1 users and $\leq 10^{-6}$ for class 2 users), we consider the values of $\Gamma_{\text {th }}^{(i)}$ obtained from Fig. 5 as the available codes in each class. For the case of no blocking (NB), as $A$ increases above zero, more packets become available with low interference. As a result, class 1 (2) throughput increases until it reaches a saturation value that is always less than the number of class 1 (2) available codes. For large enough $A$, the number of active users requesting free codes increases until there are not enough codes and no more 
users (no more interference as well) can transmit their data, and hence saturation occurs. However, at higher loads, the throughput starts to decrease, as more and more packets fail to find free codes, especially for the MLVWOOC system. When considering blocking (B) at a core node, it is seen that as $A$ increases, the blocking increases, thus limiting the throughput. From Fig. 9, class 1 throughput using MCVW-OOCs is nearly twice that using MLVWOOCs, and hence the number of supported high-QoS users is almost equal to that supported using MLVW-OOCs. Moreover, MCVW-OOC can achieve better throughput at higher offered loads. This of course is due to the reduced MAI when using MCVW-OOCs. Furthermore, it is noticed (from these two figures) that when using the correlation receiver with a hard limiter, the system throughputs of the two classes outperform those of the correlation receiver without a hard limiter. From Fig. 10, class 2 throughputs are equal for both MLVW-OOC and MCVW-OOC systems, as they support the same number of codes and have the same blocking probability [see Fig. 8(b)] in both systems. Comparing the traffic throughput from both classes, class 2 throughput outperforms that of class 1 for both MLVWOOC and MCVW-OOC systems. This of course is due to the larger number of codes supported by class 2 .

\section{Concluding Remarks}

An OMCS-GMPLS network employing MCVW-OOCs has been proposed, and its performance has been analyzed. The OMCS-GMPLS network architecture and multicode switching mechanism have been described, and the blocking probability has been obtained using a multiservice loss model. The BER, the probability of degradation, and the steady-state throughput have been derived for both correlation receivers with and without hard limiters. In our derivation, MAI has been considered as the main performance limiting factor. The performance of our OMCS-GMPLS network has been compared to the traditional OCS-GMPLS network, which employs MLVW-OOCs. We can extract the following concluding remarks.

1) The MCVW-OOC system outperforms the MLVW-OOC system in terms of BER. However, the number of supported high-QoS users is almost the same for both systems using a correlation receiver with a hard limiter.

2) The MCVW-OOC probability of degradation is less than that of the MLVW-OOC system, as the number of available codes in the MCVW-OOC system that meets the $\mathrm{BER}$ requirements is larger than that in the MLVWOOC system.

3) The MCVW-OOC system experiences a higher blocking probability than that of the MLVW-OOC system.

4) Steady-state throughput results show that the MCVWOOC system performs better than the MLVW-OOC system-specifically at higher offered loads.

\section{ACKNOWLEDGMENTS}

This work was financially supported by the Egyptian Ministry of Higher Education (MoHE).

\section{REFERENCES}

[1] S. J. B. Yoo, "Optical packet and burst switching technologies for the future photonic Internet," J. Lightwave Technol., vol. 24, no. 12, pp. 4468-4492, Dec. 2006.

[2] M. J. O'Mahoney, C. Politi, D. Klonidis, R. Nejabati, and D. Simeonidou, "Future optical networks," J. Lightwave Technol., vol. 24, no. 12, pp. 4684-4696, Dec. 2006.

[3] R. Van Caenegem, D. Colle, M. Pickavet, P. Demeester, K. Christodoulopoulos, K. Vlachos, E. Varvarigos, L. Stampoulidis, D. Roccato, and R. Vilar, "The design of an all-optical packet switching network," IEEE Commun. Mag., vol. 45, no. 11, pp. 52-61, Nov. 2007.

[4] A. Banerjee, J. Drake, J. P. Lang, and B. Turner, "Generalized multiprotocol label switching: An overview of routing and management enhancements," IEEE Commun. Mag., vol. 39, no. 1, pp. 144-150, Jan. 2001.

[5] A. Banerjee, J. Drake, J. P. Lang, and B. Turner, "Generalized multiprotocol label switching: An overview of signaling enhancements and recovery techniques," IEEE Commun. Mag., vol. 39, no. 7, pp. 144-151, July 2001.

[6] S. Huang, K. Baba, M. Murata, and K. Kitayama, "Variablebandwidth optical paths: Comparison between optical codelabelled path and OCDM path," J. Lightwave Technol., vol. 24, no. 10, pp. 3563-3573, Oct. 2006.

[7] K. Kitayama, "Code division multiplexing lightwave networks based upon optical code conversion," IEEE J. Sel. Areas Commun., vol. 16, pp. 1309-1319, Sept. 1998.

[8] K. I. Kitayama and M. Murata, "Versatile optical code-based MPLS for circuit, burst, and packet," J. Lightwave Technol., vol. 21, no. 11, pp. 2753-2764, Dec. 2003.

[9] Y. Wang and B. Li, "Optical code-labeled router based on OCDM,” J. Opt. Commun. Netw., vol. 2, no. 2, pp. 111-116, Feb. 2010.

[10] D. D. Sampson, G. J. Pendock, and R. A. Griffin, "Photonic code division multiple-access communications," Fiber Integr. Opt., vol. 16, no. 2, pp. 129-157, Mar. 1997.

[11] M. Jinno, H. Takara, B. Kozicki, Y. Tsukishima, Y. Sone, and S. Matsuoka, "Spectrum-efficient and scalable elastic optical path network: Architecture, benefits, and enabling technologies," IEEE Commun. Mag., vol. 47, no. 11, pp. 66-73, Nov. 2009.

[12] S. V. Maric, O. Moreno, and C. J. Corrada, "Multimedia transmission in fiber-optic LANs using optical CDMA," J. Lightwave Technol., vol. 14, no. 10, pp. 2149-2153, Oct. 1996.

[13] W. C. Kwong and G.-C. Yang, "Multiple-length extended carrier-hopping prime codes for optical CDMA systems supporting multirate multimedia services," J. Lightwave Technol., vol. 23, no. 11, pp. 3653-3662, Nov. 2005.

[14] E. Intay, H. M. H. Shalaby, P. Fortier, and L. A. Rusch, "Multirate optical fast frequency-hopping CDMA system using power control," J. Lightwave Technol., vol. 20, no. 2, pp. 166-177, Feb. 2002.

[15] E. Narimanov, W. C. Kwong, G.-C. Yang, and P. R. Prucnal, "Shifted carrier-hopping prime codes for multicode keying in wavelength-time O-CDMA," IEEE Trans. Commun., vol. 53, no. 12, pp. 2150-2156, Dec. 2005.

[16] C.-Y. Chang, G.-C. Yang, and W. C. Kwong, "Wavelength-time codes with maximum cross-correlation function of two for multicode-keying optical CDMA," J. Lightwave Technol., vol. 24, no. 3, pp. 1093-1100, Mar. 2006. 
[17] T. Ohtsuki, "Performance of multicode direct-detection optical CDMA systems," in Proc. IEEE GLOBECOM, 1998, pp. 3227-3232.

[18] S. V. Maric and V. K. Lau, "Multirate fiber-optic CDMA: System design and performance analysis," J. Lightwave Technol., vol. 16, no. 1, pp. 9-17, Jan. 1998.

[19] A. R. Forouzan, N.-K. Masoumeh, and N. Rezaee, "Frame time-hopping patterns in multirate optical CDMA networks using conventional and multicode schemes," IEEE Trans. Commun., vol. 53, no. 5, pp. 863-875, May 2005.

[20] J. S. Vardakas, I. D. Moscholios, M. D. Logothetis, and V. G. Stylianakis, "Performance analysis of OCDMA PONs supporting multi-rate bursty traffic," IEEE Trans. Commun., vol. 61, no. 8, pp. 3374-3384, Aug. 2013.

[21] G.-C. Yang and W. C. Kwong, "Performance comparison of multiwavelength CDMA and WDMA+OCDMA for fiberoptics networks," IEEE Trans. Commun., vol. 45, no. 11, pp. 1426-1434, Nov. 1997.

[22] G.-C. Yang, "Variable-weight optical orthogonal codes for CDMA network with multiple performance requirements," IEEE Trans. Commun., vol. 44, no. 1, pp. 47-55, Jan. 1996.

[23] H. Beyranvand and J. A. Salehi, "All-optical multi-service path switching in optical code switched GMPLS core networks," J. Lightwave Technol., vol. 27, no. 12, pp. 2001-2012, June 2009.

[24] J. S. Kaufman, "Blocking in a shared resource environment," IEEE Trans. Commun., vol. 29, no. 10, pp. 1474-1481, Oct. 1981.

[25] J. W. Roberts, "A service system with heterogeneous user requirements: Application to multi-service telecommunications systems," in Proc. Performance of Data Communications Systems and Their Applications, G. Pujolle, Ed. Holland: Amsterdam, 1981, pp. 423-431.

[26] J. A. Salehi and C. A. Brackett, "Code division multiple-access techniques in optical fiber networks-Part II: Systems performance analysis," IEEE Trans. Commun., vol. 37, pp. 834-842, Aug. 1989.

[27] M. Azizoglu, J. Salehi, and Y. Li, "Optical CDMA via temporal codes,” IEEE Trans. Commun., vol. 40, pp. 1162-1170, July 1992.

[28] H. M. Shalaby, "Optical CDMA random access protocols with and without pretransmission coordination," J. Lightwave Technol., vol. 21, no. 11, pp. 2455-2462, Nov. 2003.

[29] A. Sandouk, H. Okada, T. Yamazato, M. Katayama, and A. Ogawa, "Throughput improvement of a dual-class multicode CDMA ALOHA system with modified channel load sensing protocol," in IEEE Int. Conf. Communication (ICC), June 1999, pp. 1079-1083.

Ahmed E. Farghal received his B.S. and M.S. degrees in electrical
engineering from the Faculty of Electronic Engineering, Menufiya
University, Menouf 32952, Egypt, in 2006 and 2011, respectively.
In 2006 he joined the Electronics and Electrical Communications
Engineering Department, Menufiya University, Egypt, and was promoted to the position of Lecturer Assistant in 2011. He is currently working toward a Ph.D. degree in electrical engineering at the Graduate School of Engineering, Egypt-Japan University for Science and Technology (E-JUST), New Borg El-Arab City, Alexandria 21934, Egypt. His research interests include all-optical networks (AONs), optical CDMA, elastic optical networks, and nano-optoelectronic devices.

Hossam M. H. Shalaby (S'83-M'91-SM'99) was born in Giza, Egypt, in 1961. He received B.S. and M.S. degrees from Alexandria University, Alexandria, Egypt, in 1983 and 1986, respectively, and a Ph.D. degree from the University of Maryland, College Park, MD, USA, in 1991, all in electrical engineering. In 1991, he joined the Electrical Engineering Department, Alexandria University, and was promoted to a Professor in 2001 . He is currently on leave from Alexandria University, where he is the Acting Dean of the School of Electronics, Communications, and Computer Engineering, EgyptJapan University of Science and Technology (E-JUST), New Borg El-Arab City, Alexandria, Egypt. From December 2000 to 2004, he was an Adjunct Professor with the Faculty of Sciences and Engineering, Department of Electrical and Information Engineering, Laval University, Quebec, QC, Canada. From September 1996 to February 2001, he was on leave from Alexandria University, when he was in the following places. From September 1996 to January 1998, he was with the Electrical and Computer Engineering Department, International Islamic University Malaysia, and from February 1998 to February 2001, he was with the School of Electrical and Electronic Engineering, Nanyang Technological University, Singapore. He worked as a Consultant at SysDSoft company, Alexandria, Egypt, from 2007 to 2010. His research interests include optical communications, optical CDMA, optical burst-switching, OFDM technology, and information theory.

Prof. Shalaby has served as a Student Branch Counselor at Alexandria University, the IEEE Alexandria and North Delta Subsection, from 2002 to 2006, and served as a Chairman of the student activities committee of the IEEE Alexandria Subsection from 1995 to 1996. He received an SRC Fellowship from 1987 to 1991 from the Systems Research Center, Maryland; the State Excellence Award in Engineering Sciences in 2007 from the Academy of Scientific Research and Technology, Egypt; the Shoman Prize for Young Arab Researchers in 2002 from the Abdul Hameed Shoman Foundation, Amman, Jordan; the State Incentive Award in Engineering Sciences in 1995 and 2001 from the Academy of Scientific Research and Technology, Egypt; the University Excellence Award in 2009 from Alexandria University; and the University Incentive Award in 1996 from Alexandria University. $\mathrm{He}$ is a Senior Member of both the IEEE Photonics Society and the Optical Society (OSA).

Zen Kawasaki (M'72-SM'01) received Bachelor, Master, and Ph.D. degrees from the Graduate School of Engineering, Osaka University, Suita, Japan, in 1973, 1975, and 1978, respectively. He started his career as a Research Associate at Nagoya University, Nagoya, Japan, in 1979. He moved to Osaka University in 1989 to be a Lecturer, where he was promoted to Associate Professor and Full Professor in the Department of Electrical, Electronics and Information Engineering, Graduate School of Engineering, in 1991 and 2000, respectively. He is a Fellow of the Institute of Electrical Engineering Japan. He is currently the President of the International Committee of Atmospheric Electricity. He is also a Senior Member of the IEEE. 